ARTÍCULO ESPECIAL

\title{
Las leches infantiles durante el periodo 1955-1975 en España: años de transición e innovaciones
}

\author{
Josep Boatella* y Ricard Bou \\ Departament de Nutrició i Bromatologia, Universitat de Barcelona, Barcelona, España
}

Recibido el 14 de enero de 2011; aceptado el 25 de mayo de 2011

PALABRAS CLAVE

Dietética infantil;

Leches infantiles;

Leches en polvo;

Composición;

Historia

\section{KEYWORDS}

Infant dietetics;

Infant milk;

Powdered milk;

Composition;

History

\begin{abstract}
Resumen Siguiendo una secuencia histórica iniciada en anteriores artículos, en este trabajo se describen y analizan las características y los principales elaboradores de las leches infantiles del periodo 1955-1975 ( $n=41)$, comercializadas en España. Se observa que hay gran variabilidad en cuanto a la composición de los productos, dificultad para clasificarlos y que se utilizan ingredientes diversos en su formulación.

@ 2011 Asociación Española de Dietistas-Nutricionistas. Publicado por Elsevier España, S.L. Todos los derechos reservados.
\end{abstract}

Infant milk in Spain during the period 1955-1975: the years of transition and innovation

Abstract By following the historical sequence started in previous articles, this work describes and analyses the characteristics and producers of infant milk sold in Spain during the period from 1955 to $1975(n=41)$. A wide variability, difficulties in their classification, and the use of various new ingredients in their formulas was observed when comparing them.

(C) 2011 Asociación Española de Dietistas-Nutricionistas. Published by Elsevier España, S.L. All rights reserved.

\footnotetext{
*Autor para correspondencia.

Correo electrónico: boatella@ub.edu (J. Boatella).
} 


\section{Introducción}

En anteriores artículos ${ }^{1,2}$, se han descrito las características de las diferentes leches en polvo destinadas a la alimentación infantil comercializadas en España durante los años de la posguerra, así como los principales laboratorios que las elaboran. En este trabajo, se analizan los productos más representativos del periodo comprendido entre 1955 y 1975 , coincidente con la aparición de diversas recomendaciones de carácter internacional sobre este tipo de productos y también con la introducción de modificaciones en cuanto a su composición y elaboración.

\section{Recomendaciones e innovaciones}

Durante ese periodo (1955-1975), se produjeron importantes innovaciones en el sector de las leches infantiles, tanto desde un punto de vista nutricional como normativo y tecnológico. Quizá el elemento más destacable fue la aparición de las primeras recomendaciones para este tipo de productos, en especial las del Committee on Nutrition $(1967)^{3}$, la Food and Drug Administration $(1971)^{4}$, la Academia Americana de Pediatría (1967 y 1976) (5,6 $^{5}$ la Comisión del Codex Alimentarius $(1976)^{7}$ y, a finales de la década de los setenta, la ESPGAN ${ }^{8}$ (1977). Todas ellas habrían de contribuir de forma decisiva a la mejora de la calidad higiénica y nutricional de los productos y marcaron, sin duda, el futuro de este sector.

Por otra parte, los avances producidos en el ámbito de la pediatría y de los conocimientos sobre la composición de la leche humana y de la de vaca introdujeron nuevas exigencias y posibilidades de mejora de los productos. En este sentido, se aceptaban unos contenidos mínimos deseables de vitaminas y minerales según los criterios establecidos inicialmente por el Committee on Nutrition ${ }^{3} y$, también, que las fórmulas equilibradas debían tener un valor calórico de entre 450 y 500 por $100 \mathrm{~g}$ de leche en polvo con una distribución del 7-16\% para las proteínas, el $30-55 \%$ para las grasas y el resto para los hidratos de carbono, intervalos establecidos a partir de la diferente proporción de principios inmediatos de la leche humana $(1: 3: 7)$ y de vaca $(3: 3: 4)$.

En relación con las mejoras introducidas en la formulación de los productos, cabe destacar la utilización de nuevos ingredientes (proteínas séricas, aceites vegetales, maltodextrinas, miel, ovolecitina, etc.), el enriquecimiento en vitaminas (en especial $A, B_{1}$ y $C$ ) y en algunos minerales (hierro), así como la adición de algunos complementos como lisozima, "anfivirasa", etc.

Por lo que a los carbohidratos se refiere, debe comentarse que la necesidad de su adición con objeto de equilibrar las fórmulas presentaba algunos problemas. Los azúcares únicamente podían adicionarse a la leche a una concentración máxima del $6,5 \%$, ya que valores superiores presentaban el riesgo de aparición de dispepsias y otros trastornos ${ }^{9}$ $y$, además, si bien en principio podían utilizarse diferentes tipos de hidratos de carbono, sus distintas propiedades daban lugar a resultados también variables. En consecuencia, apareció un tema de debate relacionado con el concepto denominado el "segundo hidrato de carbono" que, por aquel entonces, incluía la lactosa, la sacarosa, las malto- dextrinas y algunos polisacáridos (alfaglucosídicos con función nutritiva y betaglucosídicos con función coloidal). Por otra parte, diferentes trabajos publicados a partir de la década de los treinta, relacionados con la utilización de miel en la alimentación infantil, motivaron que durante ese periodo aparecieran en el mercado diferentes productos (Nektarmil, Lactomiel, etc.) con este ingrediente, al que se atribuía una función positiva, no tan sólo desde el punto de vista nutritivo, sino también como bacteriostático y bactericida ${ }^{10}$. Finalmente, durante los años sesenta, algunos productos (SAM, Artiach) todavía estaban adicionados de extracto de malta que, básicamente, enriquecía las leches en vitaminas y minerales. No obstante, esta práctica desaparecería durante la década siguiente.

En relación con la fracción grasa de la leche, la presencia de ácidos grasos de cadena corta en la de vaca y las características de la humana, con una composición esta última más semejante (en cuanto a proporción de ácidos monoinsaturados y poliinsaturados) a la de algunas grasas de origen vegetal, motivaron que una de las estrategias empleadas fuera la adición de aceites vegetales a leches en polvo descremadas, con objeto de mejorar su perfil lipídico. No obstante, esta modificación introducía nuevos problemas, ya que disminuía la estabilidad del producto frente a la oxidación ${ }^{11}$ y comportaba dificultades durante su reconstitución.

En cuanto a las proteínas, cabe comentar que, con objeto de mejorar la relación albúmina:caseína de las leches en polvo (60:40 en la humana y 18:82 en la de vaca), los laboratorios Wyeth lanzaron al mercado (1962) la leche denominada SMA26 ${ }^{12}$ que, en este sentido, representó una verdadera innovación. Esta estrategia, que se generalizó rápidamente, se conseguía mediante la adición de suero desmineralizado a la leche. Todavía en relación con la fracción proteica, cabe destacar que, a mediados de la década de los setenta, empezaron a adicionarse hidrolizados de proteínas a algunas leches especiales, con objeto de mejorar el aporte en algunos aminoácidos esenciales.

Durante los años sesenta era frecuente la adición de vitaminas A, C (con objeto de compensar las pérdidas producidas durante el procesado) y $\mathrm{D}$ (prevención del raquitismo), mientras que en la década siguiente, el número de vitaminas añadidas aumentó considerablemente (grupo B, pantotenato, fólico, tocoferol, etc.), como consecuencia de la aplicación de las recomendaciones ya comentadas. En cuanto a los minerales, durante ese periodo era habitual enriquecer los productos con hierro, práctica introducida en 1955 en la leche Similac, como una modificación de su composición original, formulada por A. Bosworth en la década de los veinte.

Ya como un problema de interés tecnológico y de utilización, durante esos años apareció también el concepto de "leches instantáneas", desarrollado a partir de los trabajos de D. Peebles en 1954 e introducido por Carnation Co., que utilizaba torres de desecación para obtener leche en polvo, de esta forma se sustituyó el método de rodillos. Con ello, la leche mejoraba su solubilidad y facilidad de dispersión, en comparación con las obtenidas por los métodos tradicionales, ya que estos comportaban la formación de lactosa en estado amorfo y unas características de las partículas (tamaño y densidad) desfavorables ${ }^{13}$. En este mismo sentido, cabe comentar que la adición de lecitinas contribuyó también a la mejora de la dispersión de la leche en polvo. 


\section{Marcas y laboratorios elaboradores}

Durante las dos décadas analizadas en este trabajo aparecieron en España nuevas empresas elaboradoras de productos infantiles, algunas de ellas relacionadas con destacadas marcas europeas ya consolidadas (Dr. Wander, Milupa, Guigoz, Sandoz, etc.) y otras, con origen en nuestro país y relacionadas, la mayoría de ellas, con el ámbito médico-farmacéutico (Artiach, Faes, Ordesa, Paido, Ulta, etc.). Por otra parte, algunas de las existentes en el periodo de posguerra desaparecieron (Eles, Max, Ventosilla, etc.), mientras que Nestlé AEPA mantuvo su posición de liderazgo en el sector.

Laboratorios Artiach. Ubicados en Zaragoza, estos laboratorios habían iniciado su actividad durante los años treinta. En 1935, L. Hormaechea, químico y farmacéutico, solicitó patente de un procedimiento para la elaboración de harinas irradiadas que pronto se convertirían en su producto emblemático. Durante los años sesenta elaboraron leches en polvo (Prilac) y también complementos para la alimentación infantil (Alisol, Gludine, Eutrogen, Harinas, etc.) junto con otros productos dietéticos (Legums, Nutex, Pectilac, Pectinal, Soluba). A mediados de la década de los ochenta, Artiach cesó en la producción de este tipo de preparados ${ }^{14}$.

Faes (dietéticos infantiles Galactina). Empresa farmacéutica española (Fábrica Española de Productos Químicos y Farmacéuticos SA), creada en 1933 y ubicada en Lamiaco (Vizcaya). A comienzos de la década de los setenta elaboró algunos productos dietéticos, entre los que cabe destacar la leche en polvo denominada Lactomiel y los productos de la gama Galactina (harinas, papillas, frutas, etc. $)^{15,16}$.

Guigoz (Massanes y Grau/Nestlé). En 1968, se inauguraron, en Piloya (Asturias), unas instalaciones de la firma Massanes y Grau para la elaboración de las leches Guigoz (empresa fundada en 1855 por M. Guigoz [1868-1919] en Châtel-SaintDenis). Bajo esta marca, se comercializaron las leches Guigoz completa y semidescremada y Guigolac, denominaciones que desaparecieron del mercado tras la adquisición de estas marcas por Nestlé en 1971 y $1973^{17}$.

Milupa. Con inicios en un negocio dedicado a la fabricación de galletas, Emil Pauly (1875-1938) creó en 1930 la marca Milupa (combinación de letras de nombre y apellido), que en 1933 lanzó sus primeros productos dietéticos (harinas). En 1964, comercializó la leche infantil Milumil que, por esos años, se introdujo en España por Milupa Sociedad Distribuidora SA, y durante los años setenta, Nektarmil, Leapta, Milfarina, Mipapa, etc. En 1989, esta multinacional (Milupa AG Friedrichsdorf/Ts) adquirió la marca Nogalda SA, y en 2007, el grupo Numico, del que Milupa ya formaba parte, junto con Nutricia, Cow \& Gate, etc., pasó a integrarse a Dano$n e^{18,19}$.

Nestlé AEPA. Ya comentadas las características de la empresa en un anterior artículo ${ }^{2}$, durante ese periodo Nestlé AEPA prosiguió su expansión en el sector de leches infantiles con la elaboración de los productos Nido, Nan, Beba, Eledon, babeurre simple, Prodieton, Celac, etc.
Ordesa. En 1943, F. Cabré (industrial), J. Monné (médico), M. Bañares (farmacéutico), R. Fortuny (farmacéutico) y J. Fortuny (comercial) constituyeron la sociedad anónima Laboratorio de Especialidades Farmacéuticas y Dietéticas SA (LEFYDSA), domiciliada en Tamarite de Litera (Huesca), que el mismo año pasó a denominarse Ordesa SA. Las instalaciones estaban ubicadas en un antiguo convento (El Frari), y M. Bañares era el responsable técnico. Adquirían la leche en polvo en la cooperativa SAM y elaboraban harinas lacteadas, irradiadas, dextrovitaminadas, Nutro-Ordesa, etc. En los años 1953-1954, estos laboratorios fueron adquiridos por por J. Andreu, propietario de Infale (Hospitalet del Llobregat), entonces el director técnico era $\mathrm{E}$. Barenys. Al asociarse con F. Serra de Dalmases, la empresa instaló una fábrica en Sant Boi del Llobregat donde se inició la producción de Blevilat (12\% y 18\%). En 1973, la compañía Ordesa fue adquirida por Nutrexpa, y así se inició una nueva etapa con elaboración de productos dietéticos (Blemil, Blevit, Agluvit, Zanasec, etc.) y otros bajo licencia de las marcas Cow \& Gate y Trufood (Locasol, MCT, Galacto$\min$, etc. $)^{20,21}$.

Laboratorios Paido. Los socios Budallés (doctor en farmacia e inspector de aduanas en Port-Bou), Benejam, Garriga y Parada habían iniciado sus actividades en el sector lácteo con instalaciones en Cardedeu. En 1929 registraron distintas marcas para sus productos (Poppy, Paido, etc.) y en 1934, los primeros preparados dietéticos (leche maternizada $1,2,3)$ con el número 156 . También prepararon leches descremada, albuminosa, soupbabeurre y, con posterioridad, Dextropaido y Paico-fer. En 1945, trasladaron las instalaciones a Bellvís (Lleida) y en 1956, el negocio pasó a manos de Massagué y Vila que, a los pocos años (década de los sesenta), dejaron de preparar leches infantiles y centraron su actividad en la preparación de leches de consumo y condensadas. Las oficinas, laboratorio y almacén estaban ubicadas en Barcelona (Balmes 25 y también en Consejo de Ciento 315$)^{22}$.

Cooperativa lechera SAM (Sindicatos Agrícolas Montañeses). Comentada también en un anterior trabajo², durante ese periodo esta empresa alcanzó su mayor expansión. Durante los años cincuenta-sesenta, se comercializaron el babeurre, la leche descremada malteada, aciláctica, albuminosa y la maternizada vitaminada, y en 1972, lanzaron al mercado SAM 1 y SAM 2, de reconstitución instantánea. En 1977 se reforzó la colaboración con la Lactaria Española, que culminaría en 1979 con la creación de Lactaria Montañesa SAM del grupo LESA (Lactaria Española SA). Durante esos años, abandonó la producción de productos dietéticos, y se centró en la elaboración de leches esterilizada, condensada, en polvo, descremadas, pasteurizadas, etc. ${ }^{23}$.

Laboratorios Ulta. Fundados en 1930 por el médico aragonés $F$. Cuenca Villoro, los Laboratorios Instituto Ulta se dedicaron a la elaboración de especialidades farmacéuticas, veterinarias y productos dietéticos. Durante los años de posguerra y con la finalidad de explotar sus patentes, se creó una sociedad filial en Alemania (Deutsche Ulta $\mathrm{GmbH}$ München) y en los años setenta se constituyó Dietéticos Ulta, con objeto de separar la comercialización de especia- 
Tabla 1 Clasificación de las distintas leches infantiles comercializadas en España durante el periodo 1955-1975

\begin{tabular}{|c|c|c|}
\hline Tipo de leche & $\mathrm{n}$ & Denominación (empresa) \\
\hline Enteras & 4 & $\begin{array}{l}\text { Blevilat } 18 \% \text { (Ordesa), Guigoz compl. (Guigoz), Nido entera (Nestlé), SAM } \\
\text { compl. (SAM) }\end{array}$ \\
\hline \multicolumn{3}{|r|}{ - } \\
\hline Sin especificar & 11 & $\begin{array}{l}\text { Guigoz semidescremada, Guigolac (Guigoz), Nido, Nan, Beba (Nestlé), } \\
\text { Paicofer (Paido), Lactoanfimon, Materanfimon, Plasfimon (Ulta), } \\
\text { Adapta, Lactoveguva (Wander) }\end{array}$ \\
\hline Primer semestre & 1 & Semidescremada (SAM) \\
\hline Segundo semestre & 1 & Completa (SAM) \\
\hline Primer trimestre & 2 & Maternizada 1t (Paido), Blevilat 12\% (Ordesa) \\
\hline Segundo trimestre & 1 & Maternizada 2t (Paido) \\
\hline Tercer trimestre & 1 & Maternizada 3t (Paido) \\
\hline Ácidas & 7 & $\begin{array}{l}\text { Eledon, Pelargon, Prodieton (Nestlé), SAM babeurre y aciláctica (SAM), } \\
\text { Prilac (Artiach) }\end{array}$ \\
\hline \multicolumn{3}{|r|}{ 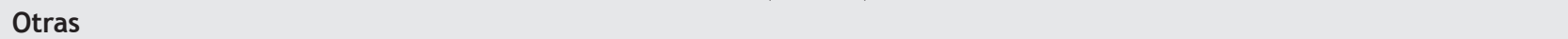 } \\
\hline Descremadas & 3 & Paido (Paido), SAM (SAM), Molico (Nestlé) \\
\hline Adición de miel & 3 & Nektarmil (Milupa), Lactomiel (Faes), Nidal (Nestlé) \\
\hline Albuminosa & 1 & Albuminosa (SAM) \\
\hline Especiales & 1 & Damira (Wander) \\
\hline
\end{tabular}

lidades farmacéuticas de la de dietéticos. Sin duda, el carácter diferencial de sus productos fue la adición, en muchos de sus preparados, de un factor de crecimiento denominado Anfivirasa ("Hallazgo científico, superación técnica, innovación alimenticia [...] principio activo de la leche fresca [...] un nuevo complejo enzimático que patentamos con el nombre de Anfivirasa"), incorporado a Anfimon, Lacto-Anfimon, Plasfimon, Materanfimon, etc., preparados que a principio de los años ochenta dejaron de elaborarse ${ }^{24}$.

Wander (Sandoz). Los trabajos de G. Wander, en 1865, sobre la utilización de la malta en la alimentación humana tuvieron continuidad con su hijo A. Wander, químico y farmacéutico, al crear la sociedad Wander. La empresa, Dr. Wander AG, tenía su sede en Berna y filiales en distintos países (The Wander Co. Inc. Chicago, p. ej.), y se dedicaba a la elaboración de productos dietéticos y farmacéuticos, entre los que destacaban los elaborados con extracto de malta, como el Maltosán (papilla preparada según los principios del Dr. Keller, discípulo de Czerny), Golma (extracto de malta cristalizado), Jemalt (preparado con aceite de hígado de bacalao), etc., la Ovomaltina, comercializada en 1904 y elaborada con leche, extracto de malta, huevos y cacao, y durante los años treinta introdujo una leche en polvo modificada con la denominación de Lactoveguva. El representante en España era J. Balarí Marco (Barcelona), hasta que se creó la filial española (SAE Wander), con J. Rius como director técnico. En 1964, inauguró una fábrica en Molins de Rei (Barcelona), destinada a la elaboración de productos dietéticos para la alimentación infantil (Lactoveguva, Nutricereal y Lacticereal). Cabe comentar que el gran éxito comercial de la Ovomaltina en otros países europeos no tuvo paralelismo en España, por lo que Wander centró su actividad en leches y papillas infantiles. Más tarde, en 1967, la firma Wander SAE fue adquirida por el consorcio farmacéutico Sandoz, y a partir de entonces se produjo el lanzamiento de las leches Adapta, Elacto y Velactín, primera leche infantil destinada a niños con alergia o intolerancia a la leche de vaca. En 1997, Sandoz se fusionó con Ciba, dando lugar a Novartis y, tras esta fusión, se creó la marca Sanutri, que desde 2006 forma parte del grupo Nutrition \& Santé25,26.

\section{Los productos}

Debido a la falta de una legislación específica, los preparados del período analizado se clasificaban de acuerdo con criterios diversos (maternizadas, humanizadas, enteras, descremadas, etc.). Así, por ejemplo, Fomon ${ }^{1}$ ) adoptaba la siguiente clasificación: a) fórmulas a base de leche de vaca (con o sin hidrato de carbono adicionado); $b$ ) acidificadas (por acción de Streptococcus lactis o por adición de ácidos láctico o cítrico); c) preparadas con leche entera, aceites vegetales e hidratos de carbono; $d$ ) leche entera desgrasada, aceites vegetales e hidratos de carbono; e) adicionadas de proteínas de suero, y $f$ ) fórmulas especiales (prematuros, alergias, errores metabólicos, etc.), con distinto valor calórico y diversos ingredientes, como proteína de soja, hidrolizados proteicos, etc. Otros autores utilizaban criterios diferentes como: a) descremadas; b) babeurres; c) semidescremadas (acidificadas o no); d) acidificadas; e) modificadas, y f) humanizadas ${ }^{27}$, criterio semejante al utilizado por Maragall: "Las leches pueden, por tanto, dividirse en leches enteras, maternizadas semidescremadas, leches acidificadas y otras, adicionadas con complementos alimenticios" 28 . Según este último criterio, se han agrupado los distintos preparados de la época $(n=41)$ cuyos denominación, tipo y fabricante se resumen en la tabla 1. 
Tabla 2 Datos de composición de los distintos tipos de leches

\begin{tabular}{lcccc}
\hline & Enteras & Maternizadas & Ácidas & Otras \\
\hline Proteína & $19,9 \pm 3,3$ & $17,7 \pm 1,6$ & $21,2 \pm 2,7$ & $22,5 \pm 2,5$ \\
Carbohidratos & $52,8 \pm 10,4$ & $58,8 \pm 5,9$ & $53,5 \pm 8,5$ & $57,3 \pm 6,1$ \\
Lípidos & $20,3 \pm 3,9$ & $17,3 \pm 6,8$ & $14,1 \pm 4,1$ & $11,7 \pm 9$ \\
Energía & $480,8 \pm 24,8$ & $485,8 \pm 11,1$ & $439,2 \pm 20,3$ & $430,7 \pm 18,8$ \\
Minerales & $4,4 \pm 1$ & $4,4 \pm 1,2$ & $4,8 \pm 1,2$ & $5 \pm 2,5$ \\
\hline
\end{tabular}

Los valores corresponden a las medianas de las leches enteras $(n=4)$, maternizadas $(n=16)$, ácidas $(n=6)$ y otras $(n=6)$. Los datos expresan media \pm desviación estándar.

Tabla 3 Número de muestras de los distintos grupos, a las que se adicionaban diferentes ingredientes

\begin{tabular}{lllll}
\hline Ingredientes & Enteras $(\mathrm{n}=4)$ & Maternizadas $(\mathrm{n}=21)$ & Ácidas $(\mathrm{n}=6)$ & Otras $(\mathrm{n}=7)$ \\
\hline Sacarosa & 3 & 10 & 3 & 1 \\
Maltodextrinas & 1 & 5 & 4 & 1 \\
Polisacáridos & 0 & 2 & 4 & 2 \\
Ácido láctico/cítrico & 0 & 0 & 6 & 0 \\
Miel & 0 & 0 & 0 & 3 \\
Aceites vegetales & 1 & 3 & 0 & 3 \\
Minerales & 0 & 5 & 1 & 1 \\
Vitaminas & 4 & 13 & 2 & 2 \\
\hline
\end{tabular}

\section{Conclusiones}

1. Durante el periodo analizado, se produjo una notable modificación del mercado de leches infantiles en nuestro país, en especial por lo que a empresas elaboradoras se refiere. Efectivamente, algunas de carácter nacional, implantadas antes o inmediatamente después de la guerra civil, cesaron en sus actividades por motivos de índole diversa (económicos, calidad y exigencias del mercado, etc.), mientras que, por el contrario, otras, con sede en diferentes países, se introdujeron en el mercado español (mediante creación de filiales, representación comercial, etc.). Cabe destacar también que durante estos años, se iniciaron procesos de fusión y adquisición que conducirían a una reducción del número de elaboradores y a la configuración de grandes empresas multinacionales.

2. Con los datos obtenidos, puede concluirse que, en general, la composición de los productos mostraba una gran variabilidad. Widdowson et $\mathrm{al}^{29}$ ya habían comentado este hecho en 1974. Si bien las denominadas leches maternizadas presentaban un aparente menor contenido en proteína y lípidos, en comparación con las leches enteras, un análisis de la varianza (ANOVA) revela, no obstante (tabla 2), que las composiciones centesimales no muestran diferencias significativas entre los cuatro grupos estudiados $(p \geq 0,05)$. La única excepción fue el valor energético $(p=0,048)$ que, de acuerdo con los contrastes de mínimas diferencias significativas (LSD; $\alpha=0,05$ ), tan sólo permite diferenciar las leches maternizadas de las clasificadas como otras leches.

3. Un análisis de los ingredientes adicionados a las fórmulas (tabla 3) muestra que las leches maternizadas y, lógica-

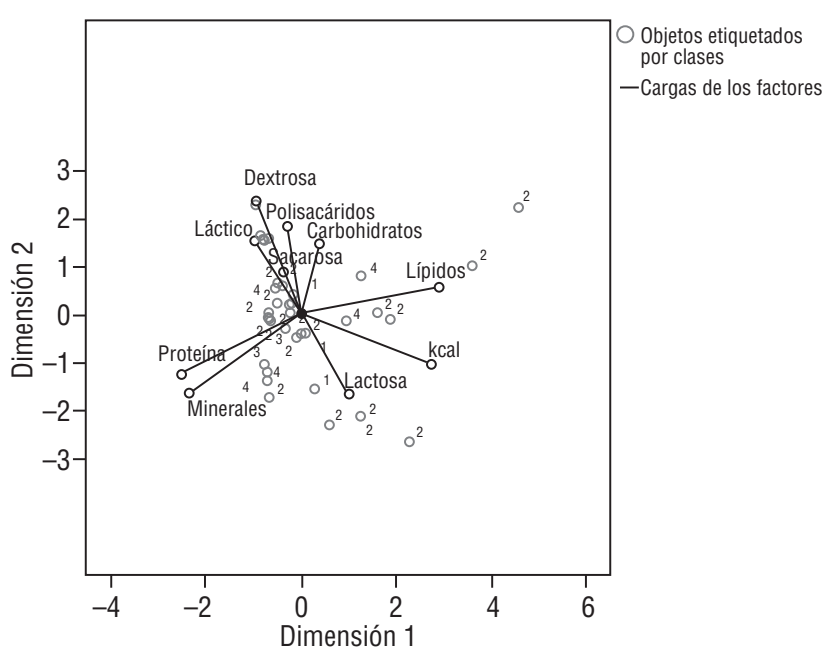

Figura 1 Análisis de los componentes principales aplicados al contenido en proteína, carbohidratos, lípidos, lactosa, sacarosa, dextrosa, polisacáridos, ácido láctico, kilocalorías y minerales considerando los cuatro tipos de leche (1: leche entera; 2: leche maternizada; 3: leches ácidas; 4: otras).

mente, las del grupo de otras leches eran las que presentaban un mayor porcentaje de adiciones y/o modificaciones. En este sentido, cabe destacar que la sacarosa fue el "segundo carbohidrato" más utilizado (en el $41 \%$ de los productos) y también que durante ese periodo se comenzó a utilizar aceites vegetales $(17 \%)$ y se generalizó el enriquecimiento en vitaminas (52\%), que incluía: $A, D, C$, E y las del grupo B. 
4. Con los datos recopilados, se realizó un análisis de los componentes principales para hallar las causas de la variabilidad de cada tipo de leche, en relación con su composición. El análisis tan sólo explica el $7 \%$ de la variabilidad de las muestras y, en consecuencia, que los cambios en la composición de las leches no obedecían a criterios de composición bien establecidos. Tal como puede observarse en la figura 1, la mayoría de las leches maternizadas se encuentran en posiciones excéntricas, en especial en los ejes correspondientes a contenido en lípidos y lactosa.

5. Las leches enteras parecen tener una composición más similar entre ellas y ocupan posiciones mucho menos excéntricas que en el caso de los demás grupos. Por otra parte, la adición de ácidos (láctico o cítrico) explica el hecho de que las muestras de este grupo se mueven principalmente en una misma dirección (fig. 1) y, finalmente, en el caso del grupo correspondiente a otras leches, se observa que las muestras también se encuentran alejadas de las posiciones centrales debido precisamente a las características de dicho grupo.

\section{Conflicto de intereses}

Los autores declaran no tener conflicto de intereses.

\section{Bibliografía}

1. Boatella J. Los primeros preparados destinados a la lactancia materna registrados en España (1915-1935). Actividad Dietética. 2009;13;173-7.

2. Boatella J, Bou R. Las leches infantiles durante la década de los años 40. Actividad Dietética. 2011 [pendiente de aceptación].

3. American Academy of Pediatrics. Committee on Nutrition. Proposed changes in food and drug administration regulations concerning formula products and vitamin-mineral dietary supplements for infants. Pediatric. 1967;40:916-22.

4. Food and Drug Administration Rules and Regulations. Label statements concerning dietary properties of food purporting to be or represented for special dietary uses. Fed Regist. 1971; 36:23553 (Part 125).

5. American Academy of Pediatrics, Committee on Nutrition. Proposed changes in food and drug administration regulations concerning formula products and vitamin-mineral dietary supplements for infants. Pediatrics. 1967;40:916-22.
6. American Academy of Pediatrics, Committee on Nutrition. Commentary on breast-feeding and infant formulas, including proposed standards for formulas. Pediatrics. 1976;57:278-85.

7. Codex Alimentarius Commission. Recomended International Standards for food for infant and children. FAO/WHO Food Standard Program. Roma; 1976.

8. Committee on Nutrition. Guidelines on infant nutrition. I. Recommendations for the composition of an adapted formula. Acta Paediatr Scand Suppl. 1977;262:1-20.

9. Beckmann R. El segundo hidrato de carbono en la alimentación artificial del lactante. Arch Pediatr. 1959;53:467-74.

10. La miel en la alimentación del lactante. Rev Esp Ped. 1965;XXI:333-40.

11. Sala JM, Albós J. Las grasas vegetales en la dietética infantil. Arch Pediatr. 1958;IX:1-9.

12. Fommon SJ. Nutrición del lactante. 2. ${ }^{a}$ ed. Madrid: Mosby/Doyma; 1995.

13. Alais C. Ciencia de la leche: principios de técnica lechera. Barcelona: Reverté; 1985.

14. Artiach Especialidades para la infancia. Zaragoza: Laboratorios Artiach; 1953.

15. Faes. Disponible en: http://www.faes.es/faes_farma/historia/0.lasso

16. Faes. Comunicación personal. Madrid; 2010.

17. La Vanguardia, 3-10-1968.

18. Millupa. Disponible en: http://www.milupa.es/nuestra-historia.php

19. La Vanguardia, 10-7-2007.

20. Ordesa. Comunicación personal. Sant Boi del Llobbregat; 2010.

21. Pérez C. Comunicación personal. Tamarite de Litera, 2010.

22. Vila N, Vila R. Comunicación personal. Bellvís; 2010.

23. Casado P, Aguayo H, Sainz F. La Cooperativa lechera SAM. Cantabria tradicional. Torrelavega; 2005.

24. Boatella J, Hernández A. Història d'un "factor de creixement": l' "Anfivirasa" (El valor i la utilitat de las hemeroteques digitals). Bull Soc Cat Hist Farm. 2010;5:7-20.

25. Morell J. Historia e historias de la industria farmacéutica barcelonesa. Butll. Soc. d'Amics de la Història i de la Ciència Farmacèutica Catalana (Quinta parte). 1997;15:27-39.

26. Hernández R. Comunicación personal. Barcelona; 2010.

27. Pérez Salamero R. La alimentación del lactante sano y con trastornos nutritivos. Actualidades en clínica y dietética infantil. Barcelona: Wander; 1972.

28. Maragall G, Maragall G, Portabella M. Guía Daimon de Farmacologia aplicada G-D 1972-73. 1. ${ }^{\text {a }}$ ed. Madrid: Daimon, Manuel Tamayo; 1972.

29. Widdowson EM, Southgate DAT, Schutz Y. Comparison of dried milk prepartions for babies on sale in 7 European countries. I. Protein, fat, carbohydrate, and inorganic constituents. Archives of Diseases in Childhood. 1974;49:867-73. 